\section{Du nouveau dans la réponse antivirale}

\section{Identification d'un régulateur négatif du facteur IFITM3}

Romain Appourchaux ${ }^{1-5}$, Andrea Cimarelli ${ }^{1-5}$
$>$ Les virus sont des «parasites » intracellulaires obligatoires ${ }^{1}$ qui se multiplient en utilisant à leur avantage le métabolisme des cellules qu'ils infectent. La réplication virale n'a cependant pas lieu dans un environnement neutre car la cellule a développé plusieurs mécanismes de défense qui sont censés combattre ces virus. Parmi celles-ci, la réponse interféron de type I (IFN-I, constitué des IFN $\alpha$ et $\beta$ ), initialement décrite en 1957 par Isaacs et Lindenmann [1], est la première ligne de défense contre les pathogènes présente dans tout le règne animal. La détection du pathogène, par le biais de plusieurs classes de récepteurs de l'immunité innée, les pattern recognition receptors (PRR), conduit à la production et la sécrétion d'IFN $\alpha / \beta$ qui, à leur tour, déclenchent une cascade de signalisation qui induit la surexpression de plusieurs centaines de gènes, communément nommés ISG (interferon stimulated genes). Ce programme génétique, profondément antiviral, résulte, entre autre, en la production de protéines ayant une activité antivirale effectrice qui sont capables d'interférer directement avec l'une ou l'autre des différentes étapes du cycle viral.

\section{Les protéines IFITM}

Les protéines de la famille IFITM (interferon-induced transmembrane proteins) et plus particulièrement les membres 1,2 et 3 , font partie de cette réponse antivirale. Ces protéines sont

\footnotetext{
${ }^{1}$ Incapables de survivre en dehors d'une cellule.
}

formées de deux domaines hydrophobes séparés par une boucle cytosolique, une structure leur permettant de s'ancrer dans les membranes lipidiques. Un effet des IFITM sur le virus de la grippe, le virus du Nil occidental (ou West Nile virus) et celui de la dengue, a été initialement décrit en 2009 par Abraham Brass par un criblage d'ARN interférence [2]. L'action antivirale des IFITM, consistant en une inhibition de la réplication virale, peut se produire de deux manières différentes. Dans une première configuration, la présence d'IFITM dans la membrane lipidique de la cellule cible inhibe l'entrée du virus en bloquant la fusion des membranes virales et cellulaires. Cette configuration est observée lors d'une activité antivirale dirigée contre plusieurs classes de virus [3] et, potentiellement, également contre des bactéries comme cela a récemment été montré pour Mycobacterium tuberculosis [4]. Dans une deuxième configuration, mise en évidence à l'heure actuelle uniquement pour le VIH-l (virus de l'immunodéficience de type 1), l'action antivirale des IFITM intervient lors des phases de production de nouvelles particules virales au sein de la cellule infectée. Dans ce cas, les IFITM sont recrutés activement, ou se retrouvent passivement, au niveau du site d'assemblage du virus et sont incorporés dans les particules virales. Les nouveaux virus ainsi produits se montrent moins infectieux et présentent un défaut dans leur capacité à entrer dans la cellule cible $[5,6]$.
${ }^{1} \mathrm{CIRI}$, international center for infectiology research, team host-pathogen interaction during lentiviral infection, université de Lyon, 46, allé d'Italie, Lyon, France ;

2 Inserm, U1111, Lyon, France ;

3 École Normale Supérieure de Lyon, 46, allé d'Italie, Lyon, France ;

${ }^{4}$ Université Claude Bernard Lyon 1, centre international de recherche en infectiologie, Lyon, France ; ${ }^{5}$ CNRS, UMR5308, Lyon, France. acimarel@ens-lyon.fr

Quelle que soit la configuration étudiée, au sein de la cellule cible ou dans la cellule productrice de virus, les mécanismes moléculaires mis en œuvre par les IFITM pour interférer avec le cycle viral ne sont pas encore bien compris [3] $(\rightarrow)$.

$(\rightarrow)$ Voir la Synthèse de K. Tartour et A. Cimarelli, $m / s n^{\circ} 4$, avril 2015, page 377

Parmi les membres

de la famille IFITM, I'IFITM3 est celui qui présente l'effet antiviral le plus systématique selon les différentes études. II reste donc un modèle de référence pour étudier la famille IFITM et ceci, même si son activité antivirale est influencée par l'identité du pathogène, le type cellulaire et la configuration. Bien que l'action d'IFITM3 soit très efficace contre de nombreux pathogènes in vitro, ces derniers sont capables de se multiplier in vivo malgré sa présence. Une des explications possibles de cette divergence est l'expression relativement faible d'IFITM3 dans les cellules qui ne sont pas stimulées par l'interféron (IFN). Cette stimulation, nécessaire à la surexpression d'IFITM3, et donc à son effet antiviral maximal, se réalise bien souvent trop tardivement (l'infection étant déjà établie) ou elle est trop faible, voire inexistante (les pathogènes ayant mis en place des mécanismes d'échappement aux récepteurs de l'immunité innée ou bloquant la réponse IFN). Trouver un moyen pour augmenter le niveau basal d'IFITM3, indépendamment de l'infection ou de la réponse IFN, constituerait donc une nouvelle piste 


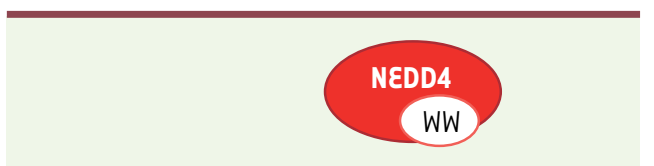

IFITM3 ${ }^{1}$ MNHTVQTFFSPVNSGQPPNYEMLKEEHEVA 30

$y:$ site de phosphorylation

$K$ : site d'ubiquitination
Figure 1. Représentation des 30 premiers acides aminés du domaine aminoterminal de la protéine IFITM3. Cette région protéique comporte des motifs importants pour la régulation post-traductionnelle de la protéine, influant ainsi sa localisation et son activité antivirale. Le premier motif YEML est un domaine d'endocytose reconnu par la sous-unité $\mu 2$ du complexe AP2. Le domaine PPNy est reconnu par le domaine WW (deux acides aminés tryptophanes séparés par environ 20 acides aminés) de l'ubiquitine ligase $\varepsilon 3$ NEDD4 permettant l'ubiquitination de la lysine en position 24 notamment [9]. La phosphorylation de la tyrosine en position 20, commune à ces deux motifs, empêche leur reconnaissance par les deux complexes. AP2 : adaptor complex 2 ; NEDD4 : neural precursor cell expressed developmentally down-regulated protein 4 . thérapeutique pour contrer un grand nombre d'infections.

\section{Comment le trafic intracellulaire} d'IFITM3 est-il régulé dans la cellule?

La localisation et le niveau intracellulaire des protéines cellulaires peuvent être finement régulés par des modifications post-traductionnelles. C'est le cas pour la protéine IFITM3. Son trafic intracellulaire est un processus dynamique et complexe qui implique différentes modifications post-traductionnelles telles que la palmitoylation, la phosphorylation, la méthylation et l'ubiquitination. Après sa synthèse dans le réticulum endoplasmique, IFITM3 transite par l'appareil de Golgi pour atteindre la membrane plasmique. IFITM3 est endocytée et se retrouve de façon prédominante, bien que non exclusive, dans les endosomes tardifs et les lysosomes.

L'étude d'une cohorte de patients hospitalisés fréquemment pour des infections respiratoires a révélé un enrichissement tout à fait remarquable, dans cette population, d'une forme mutée de la protéine IFITM3 (single nucleotide polymorphisme [SNP] rs 12252C) [7]. Le changement d'un seul nucléotide retrouvé dans cet allèle (une cytosine remplaçant une thymine) modifie l'épissage de l'ARNm codant IFITM3 et résulte en la production d'une protéine délétée de ses 21 premiers acides aminés. Contrairement à la protéine sauvage, cette IFITM3 tronquée exerce une activité antivirale amoindrie. Elle est localisée principalement au niveau de la membrane plasmique [7], suggérant que l'extrémité aminoterminale de la protéine contient des déterminants très importants pour sa localisation intracellulaire. En effet, cette portion aminoterminale qui présente le motif $y \varepsilon M L^{2}$, est reconnue par la machinerie d'endocytose dépendant d'AP2 (adaptor complex 2) (Figure 1) [3] qui est responsable de l'endocytose d'IFITM3. Cependant, le motif YعML peut aussi être phosphorylé, sur une tyrosine située en position 20, par la kinase $\mathrm{Fyn}^{3}$ bloquant la reconnaissance d'IFITM3 par le complexe AP2, ce qui conduit à la rétention d'IFITM3 à la membrane plasmique.

Un autre type de modification posttraductionnelle de IFITM3, en plus de la phosphorylation, a été mis en évidence dans une étude réalisée par le groupe de Jacob S Yount en 2012. Ces auteurs ont en effet montré la possibilité de monoet de polyubiquitination de 4 lysines présentes dans la séquence d'IFITM3, conduisant à sa dégradation. Un mutant d'IFITM3 dépourvu de ces lysines qui ne peut donc être ubiquitiné, est retrouvé en plus grande quantité dans la cellule [8]. Puisque l'activité antivirale d'IFITM3 est en partie proportionnelle au niveau de son accumulation dans la cellule, toute modification post-traductionnelle, pouvant moduler la quantité ou la stabilité d'IFITM3 au sein de la cellule, aura donc potentiellement une répercussion sur sa capacité antivirale. $\varepsilon n$ conséquence, l'identification de l'ubiquitine ligase, responsable in fine de la dégradation d'IFITM3, apparaît donc comme une étape cruciale qui

\footnotetext{
${ }^{2}$ Tyrosine-Acide glutamique-Méthionine-Leucine.

${ }^{3}$ Phospho-transférase spécifique de la tyrosine, appartenant à la famille des tyrosine protéine kinases Src, principalement localisée au niveau de la membrane plasmique et participant à la régulation de plusieurs voies de signalisation.

permettrait de réguler ce processus et ainsi d'augmenter le potentiel antiviral d'IFITM3.

\section{Identification de NEDD4}

Les récents efforts de l'équipe de Jacob S. Yount ont permis, parmi les 600 ubiquitine ligases annotées dans le génome humain, d'identifier celle qui est responsable de l'ubiquitination d'IFITM3 [9]. Ces auteurs ont étudié la famille des NEDD4 (neural precursor cell expressed developmentally down-regulated protein 4) ubiquitine ligases $\varepsilon 3$. La phosphorylation de la tyrosine située en position 20 d'IFTIM3, en plus de bloquer l'endocytose, prévient également l'ubiquitination de la protéine [10]. Or cette tyrosine 20 (y20) entre dans la constitution du motif d'endocytose YEML mais aussi dans le motif PPx ${ }^{4}$, très conservé, qui est reconnu par le domaine WW (deux acides aminés tryptophanes séparés par environ 20 acides aminés) présent sur les ubiquitines ligase $\varepsilon 3$ de la famille NEDD4, dont la protéine NEDD4 est le prototype. De nombreux indices indiquaient un rôle possible de NEDD4 dans l'ubiquitination d'IFITM3, comme son expression ubiquitaire dans les mêmes tissus, et le fait que de nombreuses cibles de NEDD4 sont en fait des protéines membranaires partageant la localisation d'IFITM3. Dans un premier temps, des expériences utilisant la microscopie confocale ont permis de confirmer la colocalisation des protéines NEDD4 et IFITM3 endogènes au niveau des lysosomes, dans des fibroblastes

\footnotetext{
${ }^{4}$ PPxy: $\mathrm{P}=$ proline, $\mathrm{x}=$ un acide aminé quelconque et $\mathrm{y}$ $=$ tyrosine.
} 


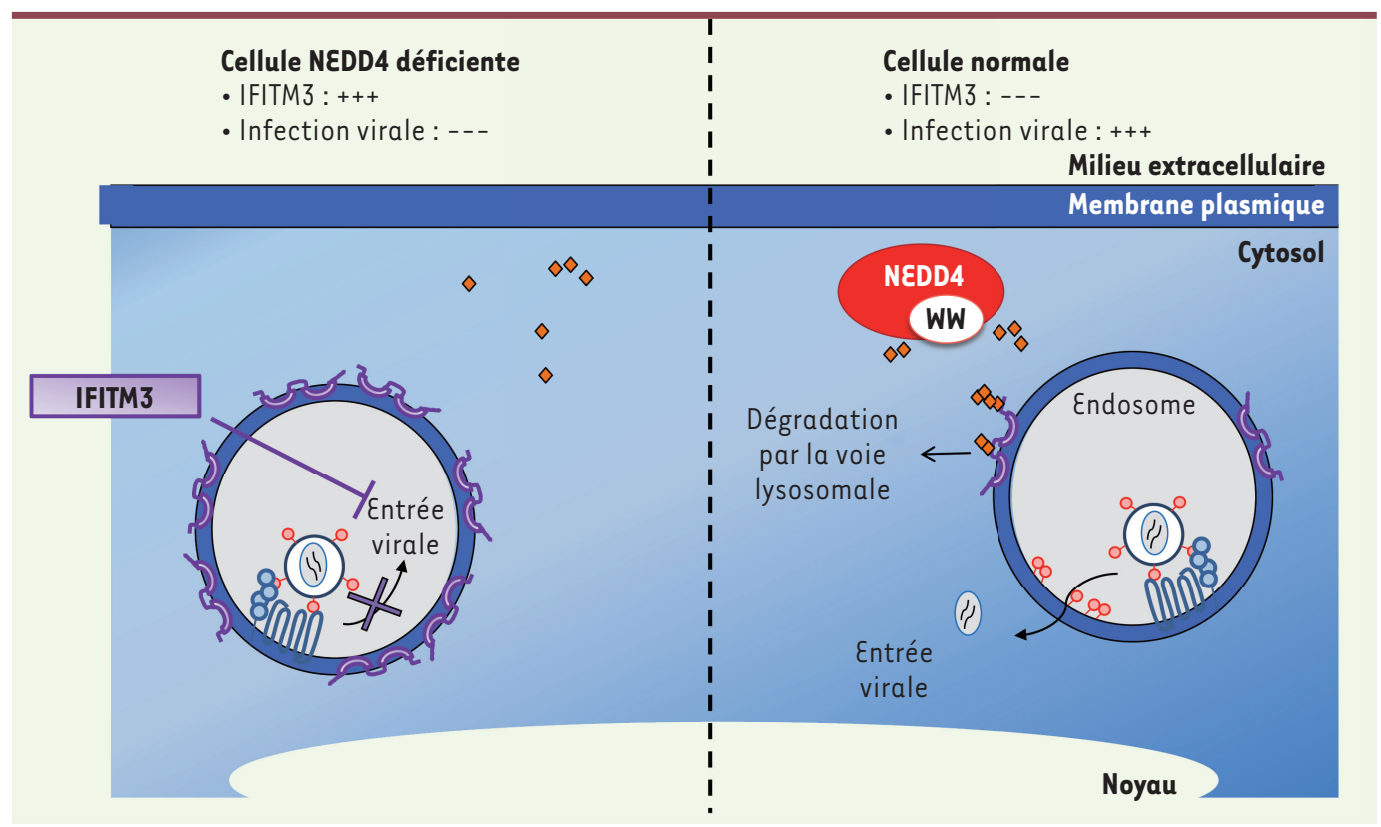

Figure 2. Régulation du niveau intracellulaire d'IFITM3 par NEDD4. Dans les cellules normales (partie de droite), I'ubiquitine ligase E3 NEDD4 reconnaît un motif PPxy de l'extrémité aminoterminale de la protéine IFITM3 (en violet) grâce à son domaine WW (deux acides aminés tryptophanes séparés par environ 20 acides aminés). Cette reconnaissance permet d'ajouter aux lysines d'IFITM3 des protéines d'ubiquitine (carré orange). Cette modification post-traductionnelle entraîne la dégradation par la voie lysosomale d'IFITM3. Le niveau intracellulaire d'IFITM3 étant faible, la réplication virale dans ces cellules est forte. Au contraire, dans les cellules déficientes en NEDD4 (partie de gauche), IFITM3 n'est pas ubiquitinée et est donc très abondante dans la membrane des endosomes. Son activité antivirale, contrant l'entrée des virus, est alors très forte et la réplication virale fortement inhibée. IFITM3 : interferon-induced transmembrane protein 3 ; NEDD 4 : neural precursor cell expressed developmentally down-regulated protein 4.

embryonnaires murins stimulés à l'interféron. Par la suite, une série d'expériences de sur-ou de sous-expression a montré l'importance de NEDD4 dans I'ubiquitination d'IFITM3 dépendant de la reconnaissance du motif PPxy. La présence de NEDD4 augmente ainsi de façon significative le niveau d'ubiquitination d'IFITM3 in cellulo mais aussi in vitro.

Les auteurs se sont également intéressés au devenir des IFITM3 ainsi ubiquitinés. NEDD4, associé aux systèmes lysosomal et endosomal, adresse des protéines vers la voie de dégradation lysosomale. L'utilisation d'un inhibiteur de l'activation des protéases lysosomales, comme la chloroquine ou la bafilomycine, augmente effectivement le niveau intracellulaire d'IFITM3 en évitant sa dégradation lorsqu'il est ubiquitiné. $\varepsilon$ n revanche, le protéasome n'est pas impliqué dans la dégradation des IFITM3 ubiquitinés. L'utilisation du MG132, inhibiteur de la voie protéasomale, n'augmente pas en effet le niveau intracellulaire d'IFITM3.

Ce travail permet également de faire le lien entre NEDD4 et l'activité antivirale
d'IFITM3 (Figure 2). L'abolition de l'expression de NEDD4 dans une lignée de cellules humaines pulmonaires permet en effet de réduire l'ubiquitination et ainsi d'augmenter le niveau intracellulaire d'IFITM3. Les cellules dépourvues de NEDD4 sont alors environ trois fois plus résistantes à l'infection par un virus Influenza A.

\section{Conclusions}

Le mécanisme antiviral d'IFITM3 n'est pas encore bien compris. Il a été suggéré qu'IFITM3 pouvait modifier le métabolisme de la cellule en influençant la fluidité membranaire afin de bloquer l'entrée du virus. Cette nouvelle étude de l'équipe de Jacob S. Yount [9] est la première à identifier un régulateur négatif cellulaire de IFITM3. La présence de NEDD4, comme régulateur négatif, semble donc indiquer qu'en l'absence d'infection virale, l'action d'IFITM3 pourrait être délétère pour le métabolisme de la cellule. $\varepsilon$ n revanche, lors d'une infection virale, la stimulation par les IFN $\alpha / \beta$ permettrait d'augmenter l'expression d'IFITM3, ce qui bloquerait la réplication virale. De manière intéressante, parmi les gènes stimulés par l'IFN (les ISG, interferon stimulated genes), on trouve le gène ISGI5 qui code une protéine qui se lie à NEDD4 et bloque son action [11]. L'augmentation du niveau intracellulaire d'IFITM3 lors de la réponse interféron résulte donc de deux mécanismes: l'augmentation de son expression et l'inhibition de sa dégradation lysosomale par NEDD4. Apparaissant naturellement lors de la réponse IFN, l'inhibition à court terme de NEDD4 est soutenue par le métabolisme celIulaire. Le développement d'inhibiteurs de NEDD4 pourrait donc potentiellement représenter une nouvelle approche préventive de lutte contre de multiples infections virales. $\diamond$

New participants in the antiviral response: identification of a negative regulator of the factor IFITM3

\section{LIENS D'INTÉRÊT}

Les auteurs déclarent n'avoir aucun lien d'intérêt concernant les données publiées dans cet article. 


\section{RÉFÉRENCES}

1. Isaacs $A$, Lindenmann J. Virus interference. I. The interferon. Proc R Soc Lond Ser B 1957 ; 147 : 258-67.

2. Brass AL, Huang IC, Benita $Y$, et al. The IFITM proteins mediate cellular resistance to influenza $A \mathrm{HINl}$ virus, West Nile virus, and dengue virus. Cell 2009 ; 139 : 1243-54.

3. Tartour K, Cimarelli A. Les IFITM, un obstacle commun à de nombreux virus. Med Sci (Paris) $2015 ; 31$ 377-82.

4. Ranjbar S, Haridas V, Jasenosky LD, et al. A role for IFITM proteins in restriction of mycobacterium tuberculosis infection. Cell Rep $2015 ; 13: 874-83$.
5. Tartour K, Appourchaux R, Gaillard J, et al. IFITM proteins are incorporated onto HIV-l virion particles and negatively imprint their infectivity. Retrovirology $2014 ; 11: 103$.

6. Compton AA, Bruel T, Porrot F, et al. IFITM proteins incorporated into HIV-1 virions impair viral fusion and spread. Cell Host Microbe 2014 ; 16 : 736-47.

7. Jia $\mathrm{R}, \mathrm{Pan} \mathrm{Q}$, Ding $\mathrm{S}$, et al. The $\mathrm{N}$-terminal region of IFITM3 modulates its antiviral activity by regulating IFITM3 cellular localization. J Virol $2012 ; 86$ : 13697-707.

8. Yount JS, Karssemeijer RA, Hang HC. S-palmitoylation and ubiquitination differentially regulate interferoninduced transmembrane protein 3 (IFITM3)-mediated resistance to influenza virus. J Biol Chem 2012 ; 287 : 19631-41.

9. Chesarino NM, McMichael TM, Yount JS. 83 ubiquitin ligase NEDD4 promotes influenza virus infection by decreasing levels of the antiviral protein IFITM3. PLOS Pathog 2015; 11 : el005095.

10. Chesarino NM, MCMichael TM, Hach JC, Yount JS. Phosphorylation of the antiviral protein IFITM3 dually regulates its endocytosis and ubiquitination. J Biol Chem 2014 ; 289 : 11986-92.

11. Malakhova OA, Zhang DE. ISG15 inhibits Nedd4 ubiquitin $\varepsilon 3$ activity and enhances the innate antiviral response. J Biol Chem 2008 ; 283 : 8783-7.

NOUVELE ${ }^{1}$ Laboratory for Pediatric Sarcoma Biology, Institute of Pathology of the LMU Munich, Thalkirchnerstrasse 36, 80337 Munich, Allemagne ;

${ }^{2}$ Institut Curie, PSL Research University, unité de Génétique

Coopération entre mutation somatique et variant génétique de susceptibilité dans le sarcome d'Ewing

Thomas G.P. Grünewald ${ }^{1-3}$, Pascale Gilardi-Hebenstreit ${ }^{4}$, Patrick Charnay ${ }^{4}$, Olivier Delattre $2,3,5$ et Biologie des Cancers, 26, rue d'Ulm, 75248 Cedex 05 Paris,

France;

${ }^{3}$ Inserm U830, Institut Curie, Centre de recherches, 26, rue d'Ulm, 75248 Paris, France ;

4 École normale supérieure, PSL Research University, Inserm U1024, CNRS UMR8197, Institut de biologie de l'ENS (IBENS), 46, rue d'Ulm, 75005 Paris, France ;

${ }^{5}$ Unité Génétique Somatique (UGS), Institut Curie, Centre hospitalier, 26, rue d'Ulm, 75248 Paris, France. olivier.delattre@curie.fr
> L'analyse génétique des cancers humains comporte, aujourd'hui, deux voies d'études principales. La première concerne l'étude des prédispositions et susceptibilités individuelles au développement de la maladie. Elle repose sur l'investigation des formes familiales de cancer et, de plus en plus, sur des approches d'association entre variants génétiques et données médicales, fondées sur l'épidémiologie génétique (en particulier les genome wide association studies, ou GWAS). L'autre voie concerne l'étude des altérations somatiques acquises par les cellules tumorales au cours de leur développement. Elle repose en grande partie sur le séquençage du génome des cellules tumorales [1]. Ainsi, pour chaque tumeur, nous disposons, d'une part, de gènes impliqués dans la susceptibilité à cette tumeur et, d'autre part, de gènes, le plus souvent distincts des premiers, impliqués dans le déve- loppement tumoral lui-même. Une question majeure est donc de comprendre la façon dont ces deux ensembles de gènes interagissent pour aboutir au développement d'un cancer donné chez un individu donné.

Certains cancers de l'enfant constituent des modèles intéressants du fait de susceptibilités individuelles assez fortes et du nombre relativement limité d'altérations somatiques. Nous avons récemment conduit une étude sur le sarcome d'Ewing et avons montré comment une mutation somatique est susceptible de coopérer avec un variant génétique constitutif pour déréguler un gène qui contribue au développement de cette tumeur [2].

\section{Le sarcome d'Ewing : une tumeur} d'origine cellulaire inconnue L'anatomo-pathologiste James Ewing identifia et décrivit, en 1921, cette tumeur éponyme comme un « endothé- liome osseux ». L'hypothèse d'une origine endothéliale que sous-entendait cette description n'a cependant pas été confirmée et la cellule à l'origine de ce sarcome reste encore inconnue $[3,4](\rightarrow)$.

$(\rightarrow)$ Voir la Nouvelle de F. Tirode et al., $\mathrm{m} / \mathrm{s} \mathrm{n}^{\circ} 3$, mars 2008 , page 248

Cliniquement, le sar-

come d'Ewing est un cancer agressif de l'enfant et du jeune adulte dont la localisation est le plus souvent osseuse.

Au niveau génétique, le sarcome d'Ewing est caractérisé par des translocations chromosomiques spécifiques, exclusivement observées dans les cellules tumorales, qui conduisent à la fusion du gène EWSRI (Ewing sarcoma breakpoint region 1 ) à différents facteurs de transcription de la famille ETS (E26 transformation-specific), le plus souvent FLII (Friend leukemia virus integration 1). Le gène de fusion résultant, $E W S R 1-F L I$, code un facteur de transcription aberrant 